\title{
Pharmacogenomics of antidepressants: what is next?
}

\author{
Molecular Psychiatry (2010) 15, 445; doi:10.1038/mp.2010.58
}

In this issue of Molecular Psychiatry, Kato and Serretti ${ }^{1}$ present a highly meticulous analysis of the state of the art in the pharmacogenomics of antidepressants. In their own words, their 'paper summarizes available literature of pharmacogenetic studies on depression from the pharmacodynamic (PD) point of view and aggregates such information into concise recommendations with meta-analysis techniques.' The major strength of this work was that the authors conducted careful meta-analysis of association studies of a series of PD variants with antidepressant treatment, having as outcome measures not only positive clinical response, but also adverse drug reactions. Their findings suggest that the serotonin transporter (both the long/short promoter polymorphism and a variable number of tandem repeats polymorphism within intron 2 or STin2), serotonin-1A and $-2 \mathrm{~A}$ receptors, tryptophan hydroxylase 1 (TPH1, which is expressed mostly in the periphery) and brain-derived neurotrophic factor (BDNF) may affect antidepressant response. Previous work by Kirchheiner et al., ${ }^{2}$ also published in Molecular Psychiatry, carefully reviewed the abundant pharmacogenetic data on the pharmacokinetics (PK) of antidepressants, including recommendations for dose adjustments based on PK parameters.

This is the 10th anniversary of the sequencing of the human genome. That led to a recent series of articles in Nature. $^{3,4}$ In one of those articles, Collins ${ }^{4}$ cites pharmacogenomics as a key advance that stems from the accomplishments of the human genome project. He states, 'Perhaps the most profound consequence of the genome revolution in the long run will be the development of targeted therapeutics based on a detailed molecular understanding of pathogenesis.'

The dream of personalized medicine always appears to be just around the corner. With all the enormous advances in technology, the possibility of cheap sequencing and genotyping and the ability to search vast amounts of data rapidly and inexpensively, we seem to be closer and closer to personalized medicine, but yet we do not seem to quite get there. Why?

Before we address that question, it would be useful to look at some background. It is absolutely obvious and self-evident that the patient's background is critical in terms of both acquiring disease and drug response. Different people exposed to the same Western diet may go on to be lean and healthy or obese and atherosclerotic. Different people exposed to the same microbial load can end up either perfectly healthy or severely infected. When different patients presenting with the same type cancer or with similar histories and clinical presentations of depression are treated with the same drug at the same doses, the results may be vastly different, ranging from full remission or even 'cure,' to terrible side effects, or to no response whatsoever, resulting in death. Every practicing doctor deals with these enormous differences in disease susceptibility and response to treatment daily and yet the science of medicine has been built mostly around standard treatments with standard doses for standard diseases. Now there is a quest to personalize therapeutics, as that would be one of the most practical and tangible outcomes of the Human Genome Project. Yet, we are stuck, and such personalization of care does not quite seem to happen in clinical medicine or psychiatry. In his Nature article, Collins ${ }^{4}$ also wrote that the success of personalized medicine will depend on continued accurate identification of genetic and environmental risk factors, and the ability to utilize this information in the real world to influence health behaviors and achieve better outcomes. This will require well-designed, large-scale research projects, for discovering risk factors and for testing the implementation of prevention and pharmacogenomic programs'.

The article by Kato and Serretti ${ }^{1}$ offers potential guidelines for future work aimed at testing the implementation of pharmacogenomic guidelines in the clinical treatment of depression. Along those lines, applied clinical research studies should be developed to test the hypothesis that genotyping for the findings that were most significant in the meta-analysis by Kato and Serretti, specifically, polymorphisms in the serotonin transporter, serotonin-1A and -2A receptors, TPH1 and BDNF genes, may serve as clinically useful guides for the treatment of depression. It is absolutely imperative that translational studies be conducted to test for the usefulness of making treatment choices based on such pharmacodynamic markers as well as on well-known PK markers in the cytochrome P450 system. If we cannot translate the outcomes of our research to real-life clinical settings, why should we be doing the research at all? It is time for pharmacogenomics to evolve from research-based promise to clinical reality in real-life settings.

J Licinio and M-L Wong
John Curtin School of Medical Research, The
Australian National University, Canberra, ACT,
Australia
E-mail: julio.licinio@anu.edu.au

\section{References}

1 Kato M, Serretti A. Mol Psychiatry 2010; 15: 473-500 (this issue).

2 Kirchheiner J et al. Mol Psychiatry 2004; 9: 442-473.

3 Venter JC. Nature 2010; 464: 676-677.

4 Collins F. Nature 2010; 464: 674-675. 